\title{
Influence of the evolving stellar X-ray luminosity distribution on exoplanetary mass loss
}

\author{
T. Penz ${ }^{1}$, G. Micela ${ }^{1}$, and H. Lammer ${ }^{2}$ \\ 1 INAF - Osservatorio Astronomico di Palermo, Piazza del Parlamento 1, 90134 Palermo, Italy \\ e-mail: [tpenz;giusi]@astropa.inaf.it \\ 2 Space Research Institute, Austrian Academy of Sciences, Schmiedlstr. 6, 8042 Graz, Austria \\ e-mail: helmut.lammer@oeaw.ac.at
}

Received 27 July 2007 / Accepted 28 September 2007

\begin{abstract}
Aims. We investigate the influence of high-energy stellar radiation at close-in orbits on atmospheric mass loss during the stellar evolution of a G-type star.

Methods. High-energy stellar luminosity varies over a wide range for $\mathrm{G}$ field stars. The X-ray luminosity distributions from the Pleiades, the Hyades, and the field are used to derive a scaling law for the evolution of the stellar X-ray luminosity distribution. A modified energy-limited escape approach is taken for calculating atmospheric mass loss for a broad range of planetary parameters. Results. We show that the evolution of close-in exoplanets strongly depends on the detailed X-ray luminosity history of their host stars, which varies over several orders-of-magnitude for G stars. Stars located in the high-energy tail of the luminosity distribution can evaporate most of its planets within $0.5 \mathrm{AU}$, while a significant fraction of planets can survive if exposed to a moderate X-ray luminosity. We show the change on an initial planetary mass distribution caused by atmospheric escape.
\end{abstract}

Key words. planetary systems - stars: activity - X-rays: stars - star: coronae

\section{Introduction}

Since the discovery of the first close-in hot Jupiter in 1995 (Mayor \& Queloz 1995) the investigation of the structure and the temporal evolution of their highly irradiated atmospheres has been a main topic for both modelers and observers. In 2002, Charbonneau et al. (2002) reported the first detection of an atmosphere around HD 209458b by observing absorption in the $\mathrm{NaI} \mathrm{D}$ lines during transits. Soon after that, Vidal-Madjar et al. $(2003,2004)$ reported the detection of several other species and pointed out that the planet is losing mass at a rate of more than $10^{10} \mathrm{~g} / \mathrm{s}$, as indicated by an expanded atmosphere likely due to heating by incoming stellar radiation. Recently, a layer of excited hydrogen atoms with a temperature of about $5000 \mathrm{~K}$ slightly above the visual radius of the planet has been reported (Ballester et al. 2007), further strengthening the hypothesis of a strongly heated atmosphere. Shortly after the discovery of hot Jupiters, theoretical models were established to verify the evaporation conditions of these close-in planets (Guillot et al. 1996). Lammer et al. (2003) used an energy-limited escape approach to investigate the atmospheric escape from close-in exoplanets. After that, several hydrodynamic models were established in order to analyze the atmospheric conditions in more detail (Yelle 2004, 2006; Tian et al. 2005; Garcia Muñoz 2007). Cecchi-Pestellini et al. (2006) have developed an accurate heat transfer model to determine the heating of planetary atmospheres due to X-rays. Mass loss calculations were also included in different models for giant planet evolution (Lecavelier des Etangs et al. 2004; Baraffe et al. 2004, 2005; Hubbard et al. 2006, 2007).
Recently, Lecavelier des Etangs (2007) presented a diagram representation where the influence of atmospheric evaporation on the evolution of the exoplanets can be estimated for a wide range of planetary parameters and for different spectral types of host stars.

However, all these works scaled the present-day solar luminosity to the considered orbital distances or, in the evolutionary models, used the scaling law derived by Guinan \& Ribas (2002) and Ribas et al. (2005) from the Sun in Time program. This program was based on a small sample of solar-type stars, allowing determination of an average scaling law for the temporal evolution of the stellar radiation for a range of wavelength from 1-1200 ̊̊. However, in reality, G stars show a broad distribution of the luminosity, which varies over a few orders of magnitude. This distribution can be observed only for the very extreme UV and X-ray (1-200 ̊) (e.g., Preibisch \& Feigelson 2005) because of interstellar absorption and the lack of sensitive instruments.

In this paper, we focus on the influence of radiation in this wavelength range, which is justified since Cecchi-Pestellini et al. (2006) showed that X-ray and very extreme UV radiation has a significant influence on planetary atmospheres. Also the X-ray luminosity of clusters with a given age like the Pleiades (Micela 2001) and the Hyades (Stern et al. 1995) is known, so that the temporal evolution of the population of $\mathrm{G}$ stars can be derived. Using this X-ray luminosity distribution as input, we apply a modified energy-limited approach formula (Erkaev et al. 2007) to study the influence of atmospheric loss for close-in exoplanets around solar-like G-stars and discuss the expected deviations from an initially known planetary mass distribution due to atmospheric loss. 


\section{Stellar X-ray luminosity distribution and temporal evolution of the radiation flux}

The Sun in Time program was established to trace the spectral irradiance of the Sun over its lifetime (Dorren \& Guinan 1994). Thus, a sample of more than 15 accurately selected solar proxies with different ages was studied. To determine the high-energy emissions this sample was reduced to 6 stars that were observed by different high-energy instruments (Ribas et al. 2005). Therefore, it is obvious that this study is limited to a small sample of accurately chosen stars, but exoplanets are orbiting a much broader range of stars, which makes it necessary to study the whole range of possible luminosities.

In fact, one of the most interesting and unexpected results of the first imaging X-ray telescopes was that stars, in particular solar-type stars, show a broad range of emission (Vaiana et al. 1981). This range is mainly due to the age-dependence of X-ray luminosity, but it has been shown that even in samples with the same age, as in open clusters, a spread of at least one order of magnitude is present (e.g., Stern et al. 1995; Micela et al. 1996). The observed spread in $L_{\mathrm{x}}$ at a fixed age is associated to the spread in rotational periods, to which the level of activity is linked (Pizzolato et al. 2003), that depends on circumstellar disk evolution in the pre-main sequence phase. In light of the discussion above, we need to consider the evolution of the complete luminosity distribution to understand the possible effects on planetary atmospheres. In this study, we focus only on the luminosity distribution of G-type stars. Because of interstellar absorption, it is not possible with present instrumentation to get information about the luminosity of a large sample of stars in the EUV (200-900 $⿱$ ), so in this work we are focusing on X-ray data. While the entire band should be considered, X-rays play, however, an important role in inducing two classes of effects. First, they modify the ionization and the chemical equilibrium of the outer planetary atmosphere, and second, they produce a significant population of secondary electrons that can penetrate down into the atmosphere, thereby contributing to its heating (Cecchi-Pestellini et al. 2006).

To get information about the temporal evolution of the X-ray luminosity we constructed a scaling law using data of stellar clusters with a known age and data from a sample of nearby solar-type stars. For a given cluster, we parameterize the logarithm of the X-ray luminosity distribution function following a log-normal distribution and then calculate the distribution for the whole sample observed in the solar neighborhood where a mix of ages exists (Schmitt 1997). The cumulative distribution function for a log-normal distribution is given as

$\operatorname{CDF}\left(\ln \left(L_{\mathrm{X}}\right)\right)=\frac{1}{2}+\frac{1}{2} \operatorname{erf}\left[\frac{\ln \left(L_{\mathrm{X}}\right)-\mu}{\sigma \sqrt{2}}\right]$,

where $\mu$ and $\sigma$ are the mean and standard deviation of the variable's logarithm. As a representative of young stars, we used the Pleiades cluster, which has an estimated age of $100 \mathrm{Myr}$ (Stauffer et al. 2005, and references therein). The maximum likelihood luminosity function of $\mathrm{G}$ stars in the Pleiades clusters is given in Micela (2002). This can be fitted well with a log-normal distribution with $\mu=67.58$ (corresponding to $L_{\mathrm{X}}=10^{29.35} \mathrm{erg} / \mathrm{s}$ ) and $\sigma=1.1$ (Fig. 1). For intermediate age stars, we used the Hyades cluster with an age of about $600 \mathrm{Myr}$ (Stauffer et al. 2005, and references therein), with the luminosity function given in Stern et al. (1995). The log-normal parameters for the Hyades are $\mu=66.8\left(L_{\mathrm{X}}=10^{29.0} \mathrm{erg} / \mathrm{s}\right)$ and $\sigma=0.9$ (Fig. 1). For $4.6 \mathrm{Gyr}$ old stars, we assumed the same standard deviation as for the

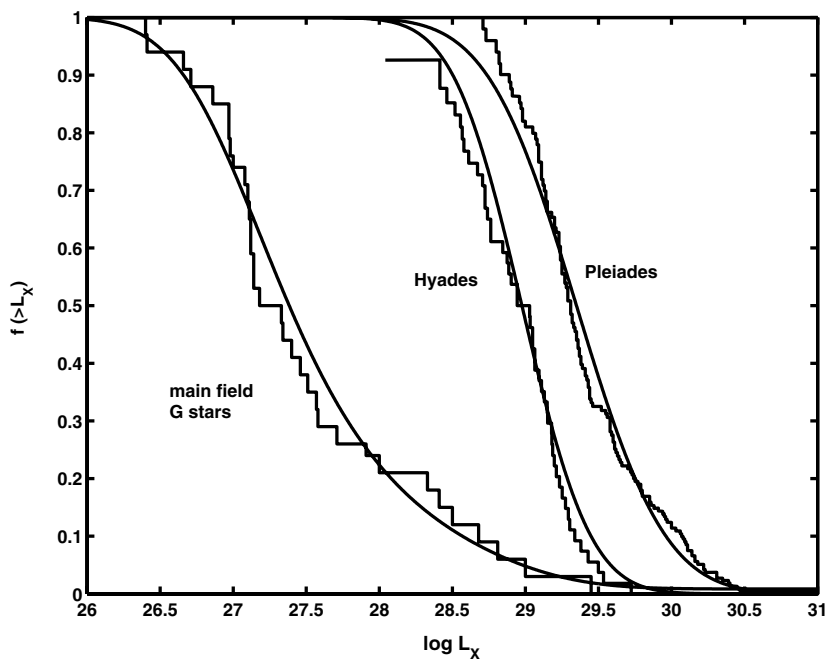

Fig. 1. Comparison between the observed and approximated cumulative X-ray distribution functions for G stars from the main field (Schmitt 1997), the Hyades (Stern et al. 1995), and the Pleiades (Micela 2002).

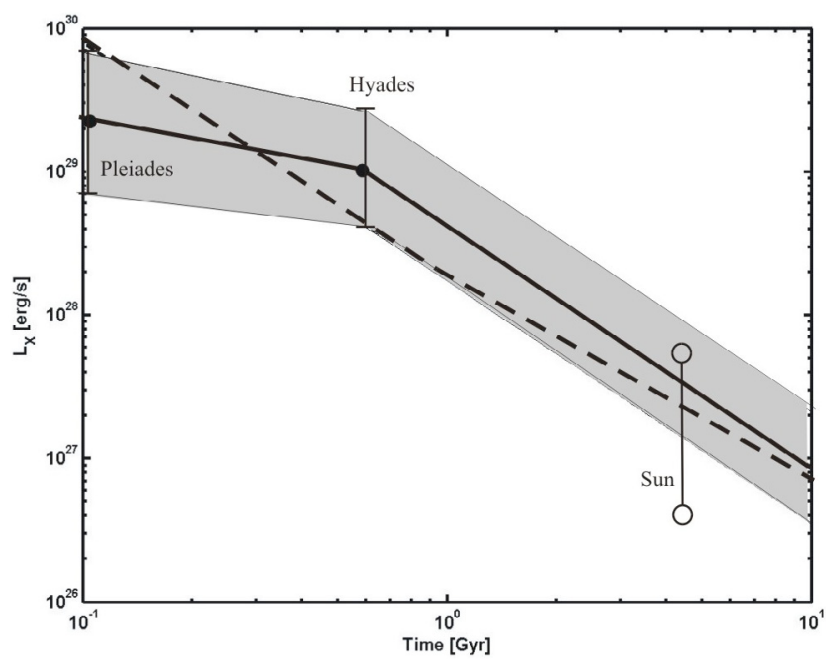

Fig. 2. $L_{\mathrm{X}}$ from Eq. (2) compared with the scaling by Ribas et al. (2005) for $1-100 \AA$ (dashed line). The shaded area gives the $1 \sigma$ equivalent derived from the log-normal distribution for the Hyades and the Pleiades. For comparison we show $L_{\mathrm{X}}$ of the Sun for maximum and minimum activity (empty circles) from Peres et al. (2000).

Hyades and a mean value of $\mu=63.3$, consistent with presentday solar emissions. Under the assumption of a constant standard deviation over time, we can derive the evolution of the total distribution using a scaling law just for the mean value according to

$L_{\mathrm{X}}=\left\{\begin{array}{cc}0.375 L_{0} t^{-0.425} & t \leq 0.6 \mathrm{Gyr} \\ 0.19 L_{0} t^{-1.69} & t>0.6 \mathrm{Gyr},\end{array}\right.$

where $L_{0}$ is the mean log luminosity of the Pleiades $\left(10^{29.35} \mathrm{erg} / \mathrm{s}\right)$. The derived scaling law is a first approximation limited by the available clusters ${ }^{1}$. In Fig. 2 we compare the scaling law from Eq. (2) with those derived by Ribas et al. (2005).

To verify the validity of the derived scaling law, we constructed the distribution function for the nearby field stars by

\footnotetext{
1 Many other clusters have been observed in X-rays, consistent with our scaling law, but not with an age greater than 600 Myr. For sensitivity reasons, it has been not possible to derive the X-ray luminosity for clusters with solar-age stars.
} 
assuming a constant stellar birth rate and a maximum age of 12 Gyr. Doing so, we can reconstruct the cumulative distribution function for G stars in Schmitt (1997) (Fig. 1) very well.

\section{Atmospheric loss calculations}

To calculate the atmospheric loss from exoplanets, we used a modified energy-limited approach, which was discussed in detail by Erkaev et al. (2007), so we shall be brief here. It is based on the work by Watson et al. (1981), who developed a model for an escaping hydrogen atmosphere, assuming that it is bound by gravity at the lower boundary, that the total incoming energy is absorbed in a narrow region where the optical depth is unity, and that the main source of heating is stellar radiation. Erkaev et al. (2007) include the influence of Roche lobe effects, which are strong at close-in distances. The planetary mass loss is given as

$\frac{\mathrm{d} M}{\mathrm{~d} t}=\frac{4 \pi R_{\mathrm{pl}}^{3} F_{\mathrm{X}}}{m M_{\mathrm{pl}} G K}$,

where we assumed for simplicity that the radius where most of the absorption takes place is close to the planetary surface. Here, $R_{\mathrm{pl}}$ and $M_{\mathrm{pl}}$ are the radius and mass of the planet, $m$ the mass of the hydrogen atom, $G$ the gravitational constant, $F_{\mathrm{X}}$ the flux at the planets orbit derived from $L_{\mathrm{X}}$, and $K$ takes Roche lobe effects into account (Erkaev et al. 2007). We can verify the formula for the case of HD 209458b, if we take $R_{\mathrm{pl}}=1.32 R_{\text {jup }}$ and $M_{\mathrm{pl}}=0.65 M_{\mathrm{pl}}$ (Knutson et al. 2007), and assume the stars luminosity to be $1.1 \times 10^{27} \mathrm{erg} / \mathrm{s}$. This value can be derived from an X-ray observation made with the XMM/Newton observatory. An X-ray source with a count rate of $3.3 \times 10^{-3} \mathrm{cts} / \mathrm{s}$ is found at the stellar position of HD 209458. Assuming a hydrogen column $N_{\mathrm{H}}=10^{19} \mathrm{~cm}^{-2}$, a coronal temperature of $T=3 \times 10^{6} \mathrm{~K}$, and a distance of $47 \mathrm{pc}$, we find $L_{\mathrm{X}}=1.1 \times 10^{27} \mathrm{erg} \mathrm{s}^{-1}$. For these values a loss rate of $1.25 \times 10^{10} \mathrm{~g} / \mathrm{s}$ is calculated, which agrees with observational limitations (Vidal-Madjar et al. 2003, 2004). One should note that the derived loss rate represents a lower limit, since it is based solely on the X-ray flux and does not consider the EUV flux from 100-900 $\AA$ in the energy budget. However, the observation by Vidal-Madjar et al. $(2003,2004)$ also presents a lower limit for the mass loss.

\subsection{Distributions for a single initial mass}

If we use the luminosity distribution described above, it is possible to calculate the mass lost from a planet for a given density and orbital distance for a certain starting mass and a given age of the host star. Because of lack of information, we assume that the density of the planet remains constant in time. Some implications of this assumption are discussed in Sect. 4. Thus, we are able to determine the distribution of planetary mass resulting from a given initial mass because of exposure to different $\mathrm{X}$-ray fluxes. It should be mentioned that we are only considering hydrogen-rich planets, meaning that we have no information about a icy/rocky core, that can remain after evaporating all the hydrogen. In Fig. 3, the mass distribution for an initial mass of $1 M_{\text {jup }}$ and densities of 0.4 (corresponding to a lowdensity planet like HD 209458b) and $1.4 \mathrm{~g} / \mathrm{cm}^{3}$ (corresponding to a high-density planet like TrES-2) and orbital distances of 0.02 and $0.05 \mathrm{AU}$ after $4 \mathrm{Gyr}$ are shown. For the closest orbit and low density, about $95 \%$ of the planets can survive, and $50 \%$ have remaining masses of more than $0.8 M_{\text {jup }}$. For a high density and an orbital distance of $0.02 \mathrm{AU}$, more than $95 \%$ have a

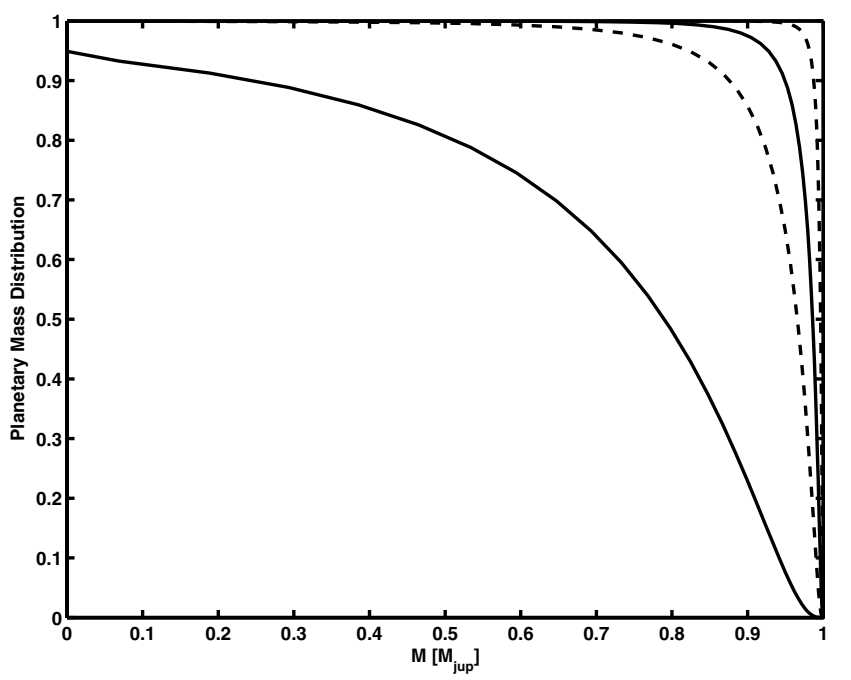

Fig. 3. Planetary mass distribution after $4 \mathrm{Gyr}$ for an initial mass of $1 M_{\text {jup }}$, densities of 0.4 (solid lines) and 1.4 (dashed lines) $\mathrm{g} / \mathrm{cm}^{3}$, and orbital distances of 0.02 and $0.05 \mathrm{AU}$ (starting from the lower curve).

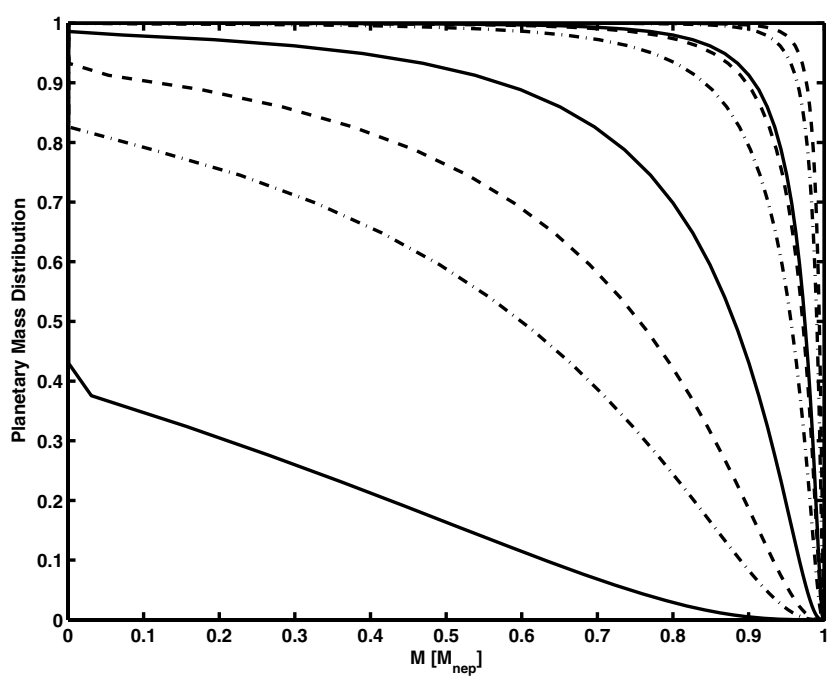

Fig. 4. Similar to Fig. 3, but for a initial mass of $1 M_{\text {nep }}$, and densities of 0.8 (solid lines) and 2.0 (dashed-dotted lines), and $3 \mathrm{~g} / \mathrm{cm}^{3}$ (dashed lines) for orbital distances of $0.02,0.05$, and $0.1 \mathrm{AU}$ (starting from the lower curve).

remaining mass of more than $0.8 M_{\text {jup }}$, while this number increases to nearly $100 \%$ for a larger orbital distance of $0.05 \mathrm{AU}$. For orbital distance $\geq 0.05 \mathrm{AU}$, only a small fraction is affected by radiation coming from stars located in the high-energy tail of the luminosity distribution. This is in agreement with predictions by Hubbard et al. $(2006,2007)$ based on a different approach that most of the hot Jupiters are not strongly influenced by mass-loss processes.

Figure 4 shows the cumulative distribution function for an initial mass of $1 M_{\text {nep }}$ for the same orbital distances but for densities of $0.8,2$ (corresponding to the density of the transiting hot Neptune observed by Gillon et al. 2007), and $3 \mathrm{~g} / \mathrm{cm}^{3}$ after 4 Gyr. At a close-in orbit about $40 \%$ of Neptune-sized planets with a density of $0.8 \mathrm{~g} / \mathrm{cm}^{3}$ can survive, while this value increases to more than $80 \%$ for a density of $2 \mathrm{~g} / \mathrm{cm}^{3}$ and to more than $90 \%$ for $3 \mathrm{~g} / \mathrm{cm}^{3}$. At an orbital distance of $0.05 \mathrm{AU}$, less than $2 \%$ of the low-density exoplanets would not survive the impact of their host star's radiation for 4 Gyr. Nearly $85 \%$ of the Neptune-mass planets orbiting at $0.02 \mathrm{AU}$ with a low 

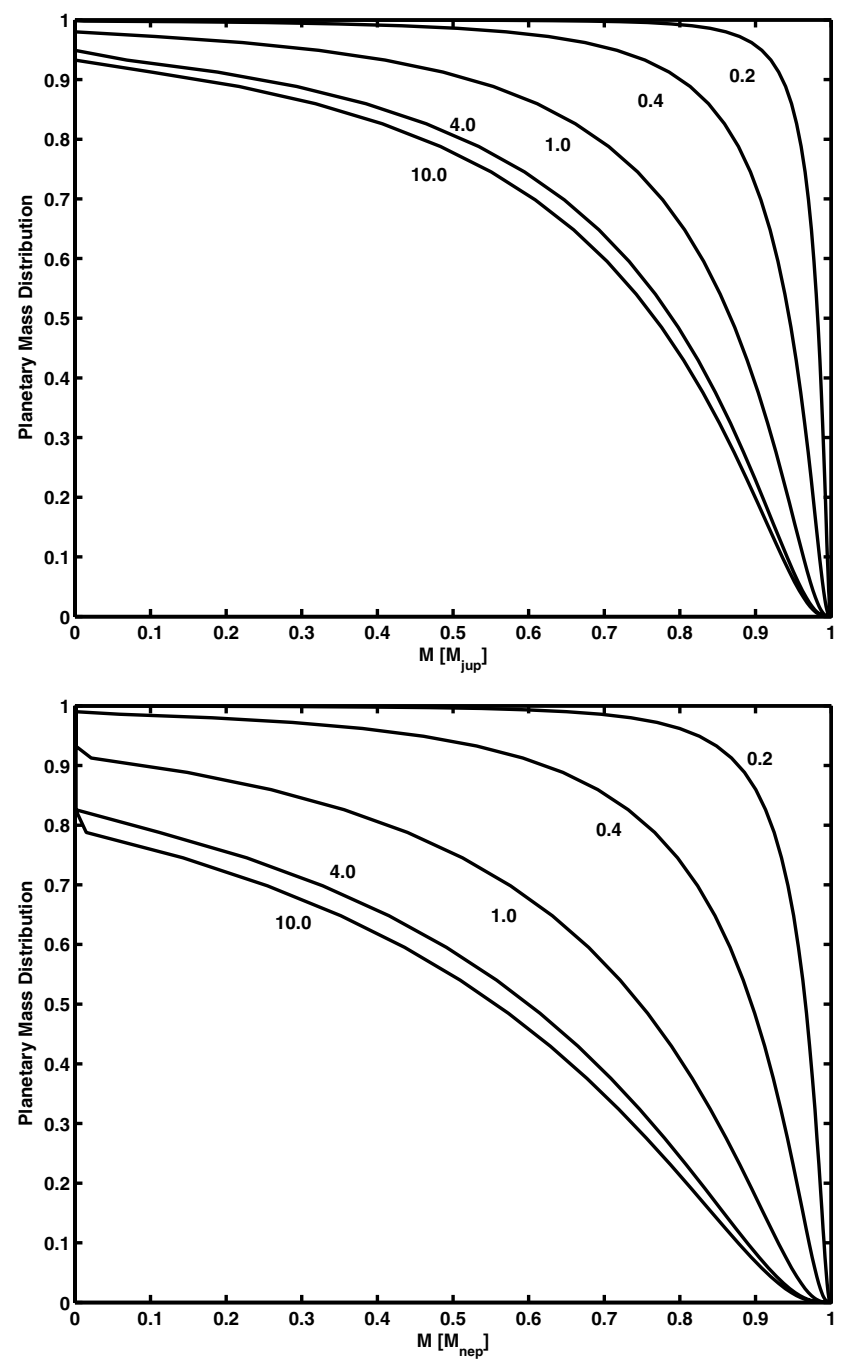

Fig. 5. Mass distribution at $0.02 \mathrm{AU}$ for a Jupiter-mass planet (upper panel, density is $0.4 \mathrm{~g} / \mathrm{cm}^{3}$ ) and a Neptune-mass planet (lower panel, density is $2 \mathrm{~g} / \mathrm{cm}^{3}$ ) at different ages of the system in Gyr.

density could be eroded to Super-Earths (about $10 M_{\oplus}$ ) and still more than $20 \%$ of the high-density planets at the same orbits. Hydrogen envelopes of $0.2 M_{\text {nep }}$ can be easily lost at the closein orbits. At $0.05 \mathrm{AU}, 30 \%$ of the low-density planets can lose such an envelope, while the number decreases to about $5 \%$ for high-density planets. At $0.1 \mathrm{AU}$, the effects are also very small for Neptune-sized planets. Stopping our calculations after $4 \mathrm{Gyr}$ is justified, which can be seen from Fig. 5, where the mass distribution at $0.02 \mathrm{AU}$ for different ages of the system is shown for Jupiter- and Neptune-mass planets, respectively. One can see that the main loss takes place in the first Gyr after the system's origin when high-energy stellar emission is higher. At later stages, the mass loss is negligible compared with the loss during early stages.

\subsection{Distributions for an initial mass distribution}

After the investigation of the atmospheric loss for a single planetary mass we proceed by evaluating the influence of loss processes on an initial distribution with different masses. Since we have no clear information of the initial mass distribution of planets formed from a circumstellar disk, we assume a flat distribution of masses ranging from $0.2 M_{\text {nep }}$ up to $10 M_{\text {jup }}$. Figure 6

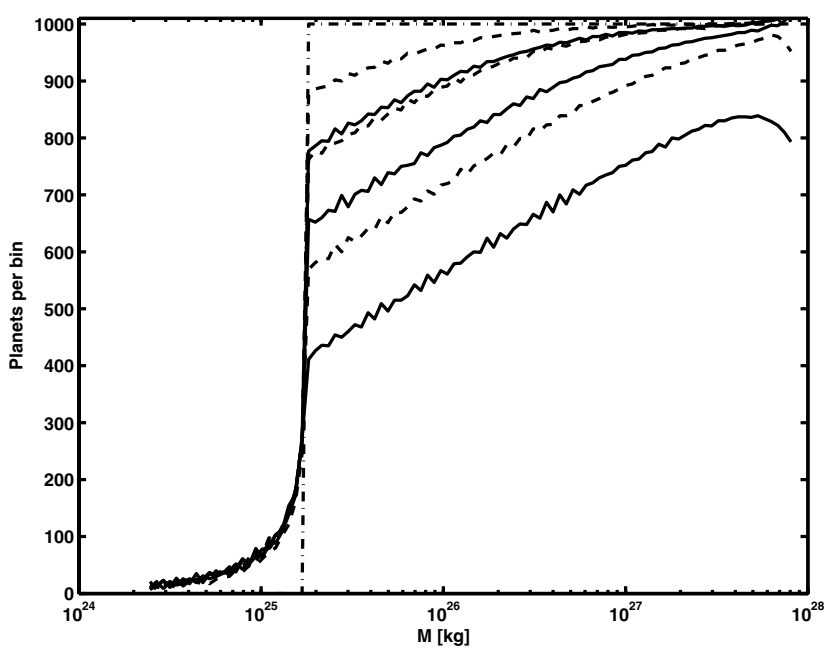

Fig. 6. Initial flat mass distribution (dotted line) and final mass distributions after $4 \mathrm{Gyr}$ for 0.4 (solid lines) and $1.4 \mathrm{~g} / \mathrm{cm}^{3}$ (dashed lines). Orbital distances are $0.02,0.05$, and $0.1 \mathrm{AU}$ (starting from the lower curve). In the low-mass tail, the distribution is similar for all cases.

shows the resulting distribution after $4 \mathrm{Gyr}$ for different orbital distances and densities of $0.4 \mathrm{~g} / \mathrm{cm}^{3}$ and $1.4 \mathrm{~g} / \mathrm{cm}^{3}$. The percentage of planets lost for $0.02,0.05$, and $0.1 \mathrm{AU}$ is $32 \%, 12 \%$, and $4 \%$, if we assume a density of $0.4 \mathrm{~g} / \mathrm{cm}^{3}$. For the higher density, the corresponding numbers are $18 \%, 5 \%$, and $1 \%$. As expected, one can see that, for the closest orbit and the lower density, most planets are lost. Furthermore, the shape of the mass distribution changes with a number of planets with masses smaller than the minimum of the initial mass function. For distances greater than $0.1 \mathrm{AU}$, the initial and the final mass distributions are similar because at these large distances the considered mass loss processes do not affect the total mass of the planet anymore and therefore do not alter the mass distribution.

\section{Discussion}

Since our work is subject to limited observational constraints, several assumptions were made. In this section we discuss the effects of some of these assumptions, in particular for Neptunetype planets where the effects are stronger. Instead of the observed luminosity distribution, an approximation by using lognormal distributions was done. As can be seen in Fig. 1, the log-normal distributions fit the observed data rather well. To check whether the choice of using a log-normal distribution function is justified, we additionally used the observed data at 0.1 and $0.6 \mathrm{Gyr}$, for which we have reliable observed luminosity functions, applied a linear interpolation in between, and compared the results for the planetary distribution function with the one achieved by using log-normal distribution functions after $0.6 \mathrm{Gyr}$ (Fig. 7). One can see that, even in the extreme case of a low-density planet in a close-in orbit, the deviation is rather small. Only for relatively unchanged masses (corresponding to the low-energy tail) there are some differences, since the lognormal distribution is slightly different from the observed luminosity distribution in this energy region. The agreement is very good for the highly eroded planets (corresponding to the highenergy tail). For a less extreme case, the curves are rather similar. Thus we assume that the use of log-normal distribution functions instead of the observed values does not introduce significant errors. 


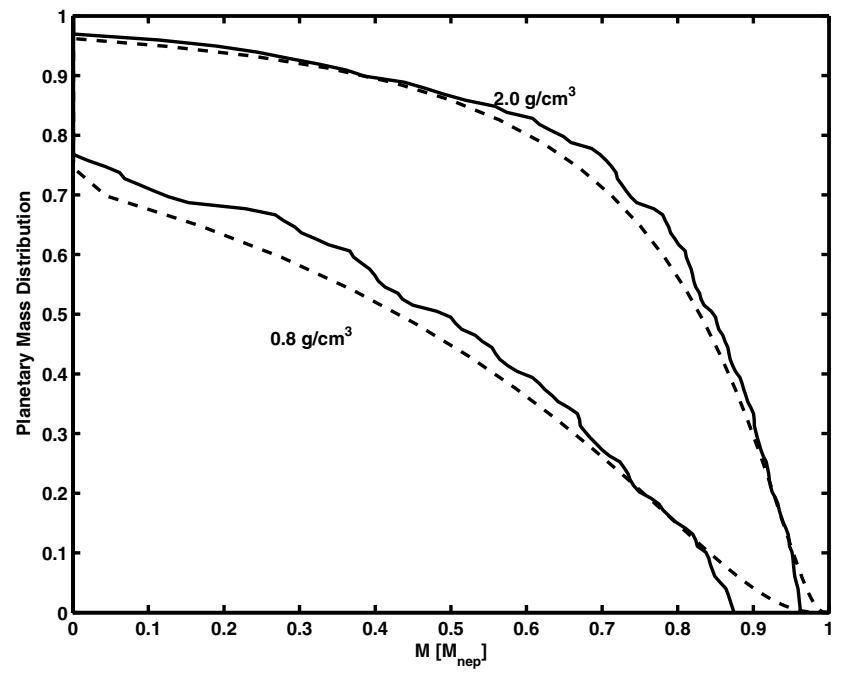

Fig. 7. Planetary mass distribution after 0.6 Gyr using a linear interpolation between the observed luminosity distribution functions of the Pleiades and the Hyades (solid lines) and using log-normal distribution functions (dashed lines) for two different densities and a Neptune-sized planet at $0.02 \mathrm{AU}$.

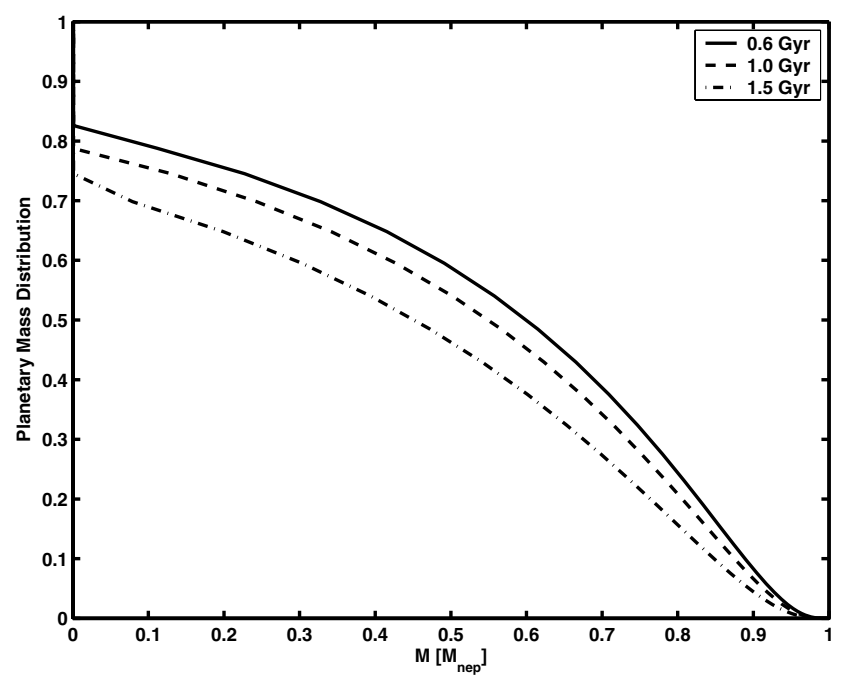

Fig. 8. Planetary mass distribution after $4.6 \mathrm{Gyr}$ for different timing of the transition between the two scaling laws in Eq. (2) for a Neptunesized planet with a density of $2 \mathrm{~g} / \mathrm{cm}^{3}$ at $0.02 \mathrm{AU}$.

Other uncertainties pertain to the scaling law used in Eq. (2). The behavior of the X-ray luminosity over the first $0.6 \mathrm{Gyr}$ is well known (Micela 2002) but there are no data available for older ages. It might be possible that the time for the transition between the two scaling laws is not $0.6 \mathrm{Gyr}$ but some older age. However, it cannot be older than $1.5 \mathrm{Gyr}$, because for older ages we cannot reconstruct the observed luminosity distribution function from Schmitt (1997) anymore. The effect of shifting the transition time to older ages on the planetary mass distribution is shown in Fig. 8. For $t \leq 1 \mathrm{Gyr}$, the difference is less than 5\%, and even for the rather unlikely case of $t=1.5 \mathrm{Gyr}$ the error is less than $10 \%$.

In our study, we used the simplification that the density of the planets remains constant over time. Lecavelier des Etangs (2007) derives a simple scaling law for the radius of exoplanets with $M_{\mathrm{p}} \geq 0.1 M_{\text {jup }}$. Using this scaling law it is possible to derive a scaling law for the temporal evolution of the density as $1 /\left(1+\beta t^{-0.3}\right)^{3}$, where $\beta=0.2$ for $M_{\mathrm{p}} \geq 0.3 M_{\text {jup }}$. We



Fig. 9. Planetary mass distribution after 4.6 Gyr using a constant density (solid line) and for the scaling law derived by Lecavelier des Etangs (2007) (dashed lines) for a Jupiter-sized planet with a density of 0.4 and $1.4 \mathrm{~g} / \mathrm{cm}^{3}$ at $0.02 \mathrm{AU}$.

normalized this density to a density of 0.4 and $1.4 \mathrm{~g} / \mathrm{cm}^{3}$ for a planet with one Jupiter mass and an age of 4 Gyr (Fig. 9). One can see that this effect has more impact compared with the uncertainties discussed before. For a low-density hot Jupiter, it gives a difference of 10-20\% compared with the case of a constant density. The larger loss of planets is caused by the effect that, according to this scaling law, the initial density is lower by about a factor of 2 . As expected, the effect is less pronounced for planets with higher densities.

The derived mass function could be compared with the observed mass distribution of known planets. However, we prefer to avoid comparing our predictions with the present-day planetary mass distribution because of the complex biases present in the observed sample. A major bias stems from the lack of active stars in the observed sample. Indeed, active stars are eliminated from samples since the high activity of the star makes detecting a planet more difficult. On the other hand, the effect studied here is particularly relevant for the most active stars with large X-ray emissions. We expect that CoRoT will give us a sample of planet without biases against activity for which it will be possible to check our predictions.

\section{Conclusions}

We studied the influence of the stellar luminosity distribution on the atmospheric escape of exoplanets at orbits smaller than $0.1 \mathrm{AU}$. We showed that a significant amount of planets can be evaporated over time scales of $4 \mathrm{Gyr}$ and that the final mass distribution is different from the initial one. For a Jupiter-mass planet with a density of $0.4 \mathrm{~g} / \mathrm{cm}^{3}$, about $5 \%$ of the planets are lost after 4 Gyr at an orbit of 0.02 AU. For Neptune-mass planets, this number increases to about $60 \%$ for a density of $0.8 \mathrm{~g} / \mathrm{cm}^{3}$. We also show that, for close-in orbits, a large number of planets can be eroded to Super-Earth and/or may lose dense hydrogen envelopes. Furthermore, we present the resulting mass distribution of an initially flat mass distribution of planets between $0.2 M_{\text {nep }}$ and $10 M_{\text {jup }}$. For close-in orbits, about $32 \%$ of the initial planets are lost and the distribution is shifted to smaller masses. 
Our work is a first step toward understanding the evolution of planetary mass distribution caused by the stellar activity evolution. Our approach is subject to several assumptions that will be verified with future observations and/or modeling. First of all we assume a constant density in time and a heating function of $100 \%$. Better knowledge of the densities of exoplanets will be achieved by further modeling supported by observational evidence, while the latter assumption needs to be verified with detailed radiative transfer calculations. The scaling law of the $L_{\mathrm{X}}$ evolution in Eq. (2) is derived from a few points. This potential source of uncertainty is mitigated, however, by the fact that most of the effects occur in the first Gyr (see Fig. 5) where the X-ray evolution is better known. The UV flux and evolution should be added in the future. We plan to extend our work to lower-mass stars where the X-ray effects may influence the habitability zone.

Acknowledgements. We thank the referee for useful comments, that helped to improved the paper substantially. This work is supported by the Marie Curie Fellowship Contract No. MTKD-CT-2004-002769 of the project "The influence of stellar high radiation on planetary atmospheres", and the host institution INAF-Osservatorio Astronomico di Palermo. Also acknowledged is support by ASI-INAF contract I/088/06/0. The authors also thank the Austrian Ministry bm:bwk and ASA for funding the CoRoT project.

\section{References}

Ballester, G. E., Sing, D. K., \& Herbert, F. 2007, Nature, 445, 511

Baraffe, I., Selsis, F., Chabrier, G., et al. 2004, A\&A, 419, L13

Baraffe, I., Chabrier, G., Barman, T. S., et al. 2005, A\&A, 436, L47

Cecchi-Pestellini, C., Ciaravella, A., \& Micela, G. 2006, A\&A, 458, L13

Charbonneau, D., Brown, T. M., Noyes, R. W., \& Gilliland, R. L. 2002, ApJ, 568,377

Dorren, J. D., \& Guinan, E. F. 1994, in The Sun as a Variable Star: Solar and Stellar Irradiance Variations, ed. J. M. Pap, C. Frolich, H. S. Hudson, \& S. Solanki, Proc. IAU Colloq., 143, 206
Erkaev, N. V., Lammer, H., Kulikov, Yu N., et al. 2007, A\&A, 472, 329

Garcia Muñoz, A. 2007, Planet. Space Sci., 55, 1426

Gillon, M., Pont, F., Demory, B.-O., et al. 2007, A\&A, 472, L13

Guinan, E. F., \& Ribas, I. 2002, in The Evolving Sun and its Influence on Planetary Environments, ed. B. Montesinos, A. Gimenez, \& E. F. Guinan, ASP Conf. Proc., 269, 85

Guillot, T., Burrows, A., Hubbard, W. B., Lunine, J. I., \& Saumon, D. 1996, ApJ, 495, L35

Hubbard, W. B., Hattori, M. F., Burrows, A., Hubeny, I., \& Sudarsky, D. 2006, Icarus, 187, 358

Hubbard, W. B., Hattori, M. F., Burrows, A., \& Hubeny, I. 2007, ApJ, 658, L59

Knutson, H. A., Charbonneau, D., Noyes, R. W., Brown, T. M., \& Gilliland, R. L. 2007, ApJ, 655, 564

Lammer, H., Selsis, F., Ribas, I., Guinan, E. F., \& Bauer, S. J. 2003, ApJ, 598, L121

Lecavelier des Etangs, A. 2007, A\&A, 461, 1185

Lecavelier des Etangs, A., Vidal-Madjar, A., McConnell, J. C., \& Hébrard, G. 2004, A\&A, 418, L1

Mayor, M., \& Queloz, D. 1995, Nature, 378, 355

Micela, G. 2002, in The Evolving Sun and its Influence on Planetary Environments, ed. B. Montesinos, A. Gimenez, \& E. F. Guinan, ASP Conf. Proc., 269, 263

Micela, G., Sciortino, S., Kashyap, V., Harnden Jr., F. R., \& Rosner, R. 1996, ApJS, 102, 75

Peres, G., Orlando, S., Reale, F., Rosner, R., \& Hudson, H. 2000, ApJ, 528, 537 Pizzolato, M., Maggio, A., Micela, G., Sciortino, S., \& Ventura, P. 2003, A\&A, 397,147

Preibisch, T., \& Feigelson, E. D. 2005, ApJS, 160, 390

Ribas, I., Guinan, E. F., Güdel, M., \& Audard, M. 2005, ApJ, 622, 680

Schmitt, J. H. M. M. 1997, A\&A, 318, 215

Stauffer, J. R., Rebull, L. M., Carpenter, J., et al. 2005, ApJ, 130, 1834

Stern, R. A., Schmitt, J. H. M. M., \& Kahabka, P. T. 1995, ApJ, 448, 683

Tian, F., Toon, O. B., Pavlov, A. A., \& De Sterck, H. 2005, ApJ, 621, 1049

Vaiana, G. S., Cassinelli, J. P., Fabbiano, G., et al. 1981, ApJ, 245, 163

Vidal-Madjar, A., Lecavalier des Etangs, A., Désert, J.-M., et al. 2003, Nature, 422,143

Vidal-Madjar, A., Désert, J.-M., Lecavelier des Etangs, A., et al. 2004, ApJ, 604, L69

Watson, A. J., Donahue, T. M., \& Walker, J. C. G. 1981, Icarus, 48, 150

Yelle, R. 2004, Icarus, 170, 167

Yelle, R. 2006, Icarus, 183, 508 